Acta Crystallographica Section F

Structural Biology

and Crystallization

Communications

ISSN 1744-3091

\section{Preliminary crystallographic analysis of the antibiotic discharge outer membrane lipoprotein OprM of Pseudomonas aeruginosa with an exceptionally long unit cell and complex lattice structure}

Crystals of the drug-discharge outer membrane protein OprM $(\mathrm{MW}=50.9 \mathrm{kDa})$ of the MexAB-OprM multidrug transporter of Pseudomonas aeruginosa have been grown at $293 \mathrm{~K}$ in the presence of 2-methyl-2,4-propanediol and a combination of surfactants. The crystal belonged to space group $R 32$, with unitcell parameters $a=b=85.43, c=1044.3 \AA$. Diffraction data for OprM were obtained using the undulator synchrotron-radiation beamline at SPring-8 (BL44XU, Osaka University), which allowed an extra-long specimen-todetector distance with a wide detector area. The crystal diffracted to $2.56 \AA$ resolution using $0.9 \AA \mathrm{X}$-rays from the synchrotron-radiation source. A heavyatom derivative for isomorphous replacement phasing was obtained using iridium chloride.

\section{Introduction}

Xenobiotic/antibiotic efflux transporters are ubiquitous to all living organisms and protect cells from small noxious compounds. When such transporters are expressed in neoplastic cells, they render the cancer cells resistant to chemotherapeutic agents; in bacteria, they render cells resistant to many structurally and functionally dissimilar antibiotics (termed multidrug-resistance). Development of agents that inhibit the function of the multi-antibiotic efflux pump and can shift back antibiotic resistant cells to being susceptible, lower the antibiotic dosage and thus lower the adverse effect of antibiotics is awaited. A short-cut to this goal may be protein structure-based drug design and development.

The OprM (MW $=50.9 \mathrm{kDa} ; 468$ amino acids) subunit of the MexAB-OprM efflux pump of Pseudomonas aeruginosa is a lipoprotein-type outer membrane protein that is assumed to form a transmembrane drug-discharge duct across the outer membrane and to facilitate antibiotic disposal (Nakajima et al., 2000). Thus, it is of interest to reveal the structure of the drug-disposal duct and to determine whether OprM possesses multiple modes of membrane anchoring. The structure of OprM may differ from that of the porinlike open duct, as the protein facilitates the exit of antibiotics but not the entry. The cognate partners of the OprM subunit in the pump assembly are the intrinsic inner membrane protein MexB and the periplasmic MexA subunit, which serve as a drug-recognition/energyconversion subunit and a molecular clamp connecting the MexB and OprM subunits, respectively.

MexAB-OprM belongs to the resistance-nodulation cell-division (RND) superfamily assembly. The structures of three proteins from this superfamily have been reported to date. The structure of AcrB, a MexB homologue, has been resolved at 2.7-3.5 $\AA$ resolution and was revealed to mainly consist of three domains: a transmembrane domain, an extra-membranous channel/vestibule domain and a funnel domain (Murakami et al., 2002; Pos et al., 2004). Of the membrane-fusion family proteins (MFP), the structure of MexA has been solved at 2.40-3.00 $⿱$ resolution, which showed a long sickleshaped protein mainly consisting of four domains: an $\alpha$-helical hairpin domain, a $\beta$-domain consisting of eight $\beta$-sheets, a third domain containing seven $\beta$-sheets and a short $\alpha$-helix and an addi- 
Table 1

Data-collection statistics.

Values in parentheses are for the highest resolution shell.

\begin{tabular}{|c|c|c|c|}
\hline & Native-1 & Native-2 & $\mathrm{IrCl}_{3}$ derivative \\
\hline Data collection & BL44XU & BL44XU & BL44XU \\
\hline Wavelength (§) & 0.9000 & 0.9000 & 1.1056 \\
\hline Resolution $(\AA)$ & $40.0-2.56(2.69-2.56)$ & $40.0-2.95(3.06-2.95)$ & $40.0-3.30(3.42-3.30)$ \\
\hline Space group & $R 32$ & $R 32$ & $R 32$ \\
\hline \multicolumn{4}{|l|}{ Unit-cell parameters $(\AA)$} \\
\hline$a=b$ & 85.43 & 84.92 & 85.31 \\
\hline$c$ & 1044.3 & 1042.6 & 1043.0 \\
\hline Observed reflections & 548330 & 471037 & 322693 \\
\hline Independent reflections & 48709 & 30962 & 41952 \\
\hline Percentage of rejected reflections (\%) & 0.05 & 1.34 & 0.56 \\
\hline$R_{\text {merge }} \dagger$ & $0.047(0.362)$ & $0.069(0.432)$ & $0.056(0.227)$ \\
\hline Completeness (\%) & $99.1(94.5)$ & $97.5(99.9)$ & $99.0(100.0)$ \\
\hline Multiplicity & 11.4 & 15.2 & 7.7 \\
\hline$I / \sigma(I)$ & $6.4(2.0)$ & $35.5(8.7)$ & $31.7(8.8)$ \\
\hline
\end{tabular}

$\dagger R_{\text {merge }}=\sum \sum_{j}\left|\langle I(h)\rangle-I(h)_{j}\right| / \sum \sum_{j}\langle I(h)\rangle$, where $\langle I(h)\rangle$ is the mean intensity of symmetry-equivalent reflections. Friedel pairs were merged as individual data.

tional disordered domain (Akama et al., 2004; Higgins et al., 2004). Of the outer membrane family proteins, the structure of TolC has been solved at $2.1 \AA$ resolution and consisted of two major domains: an outer membrane-spanning $\beta$-barrel and a long periplasmic $\alpha$-barrel (Koronakis et al., 2000). Both ends of the barrel had pore openings. OprM differs from TolC in that the protein participates solely in xenobiotic discharge, whereas TolC is a multifunctional outer membrane protein involved in protein secretion and xenobiotic extrusion; OprM also has N-terminal fatty-acid modification but TolC does not. Therefore, it is important to clarify the structure of this medically relevant pro-xenobiotic discharge protein of the RNDefflux pump.

\section{Preparation of OprM}

P. aeruginosa TNP072 cells lacking the chromosomal oprM harboured pMMB67EH, which carries the oprM-(his) ${ }_{6}$ fusion gene (Nakajima et al., 2000). Cells were grown overnight in $800 \mathrm{ml}$ Luria broth supplemented with $50 \mu \mathrm{g} \mathrm{ml}^{-1}$ sulbenicillin at $310 \mathrm{~K}$ under constant rotation at $200 \mathrm{rev} \mathrm{min}^{-1}$. The culture was diluted with $9200 \mathrm{ml}$ Luria broth and rotation continued as above; isopropyl- $\beta$-Dthiogalactoside (IPTG; Wako Pure Chemical Industries Ltd) was then added to $1 \mathrm{~m} M$ when $A_{600}^{1 \mathrm{~cm}}$ reached 0.7 and the flask was rotated for an additional $3 \mathrm{~h}$. Cells were harvested by centrifugation at $10000 \mathrm{~g}$ for $10 \mathrm{~min}$ at $283 \mathrm{~K}$, washed once with $100 \mathrm{ml}$ of a solution containing $20 \mathrm{~m} M$ sodium phosphate buffer with $150 \mathrm{mM} \mathrm{NaCl} \mathrm{pH}$ 7.2 and suspended in $45 \mathrm{ml}$ of the same buffer containing one tablet of protease-inhibitor mixture (Complete Mix, Roche Applied

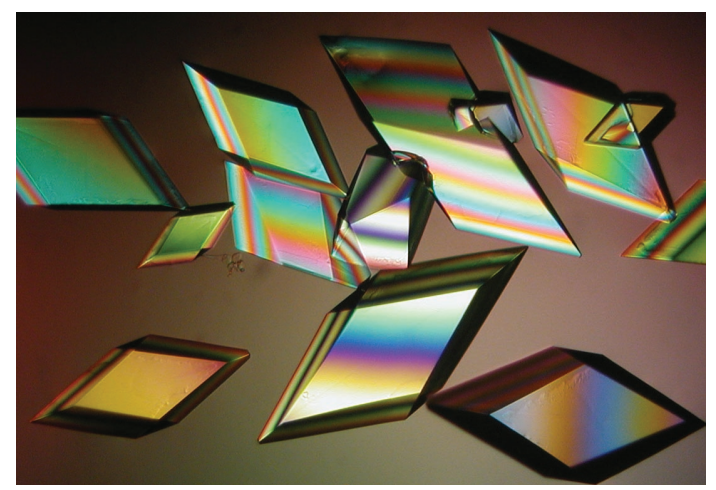

Figure 1

Crystals of native OprM from $P$. aeruginosa.
Science) and about $5 \mu \mathrm{g} \mathrm{ml}^{-1}$ of DNaseI (Worthington Biochemical Corp.). Cells were disintegrated by passing them three times through a French pressure cell at $82.7 \mathrm{MPa} \mathrm{cm}^{-2}$ and unbroken cells were removed by centrifugation at $10000 \mathrm{~g}$ for $15 \mathrm{~min}$ at $277 \mathrm{~K}$. The supernatant fraction was subjected to high-speed centrifugation at $150000 \mathrm{~g}$ for $60 \mathrm{~min}$ at $277 \mathrm{~K}$, the pellets were washed once by centrifugation under the same conditions as above and were suspended in $80 \mathrm{ml} 20 \mathrm{~m} M$ sodium phosphate buffer $\mathrm{pH}$ 7.2. Freshly prepared $N$-lauryl sarcosine solution ( $N$-dodecanoyl- $N$-methylglycine sodium salt; $\mathrm{MW}=293.4$; nakalai tesque Corp.) was added to $0.8 \%(w / v)$ and the mixture was incubated at $277 \mathrm{~K}$ for 20 min under constant stirring and then centrifuged at $150000 \mathrm{~g}$ for $60 \mathrm{~min}$ at $277 \mathrm{~K}$. The pellets were washed with $80 \mathrm{ml} 20 \mathrm{mM}$ sodium phosphate buffer pH 7.2 supplemented with $5 \%(v / v)$ glycerol and were suspended in $50 \mathrm{ml}$ of $20 \mathrm{mM}$ sodium phosphate, $20 \mathrm{mM}$ imidazole, $2.5 \%(w / v) n$-octyl- $\beta$-D-glucopyranoside (Octyl-Glc; CMC $=19 \mathrm{mM}$, $\mathrm{MW}=292.4$; Alexis Corp.) and $0.3 \mathrm{M} \mathrm{NaCl}$. The mixture was gently stirred at $277 \mathrm{~K}$ for $30 \mathrm{~min}$ and centrifuged at $150000 \mathrm{~g}$ for $60 \mathrm{~min}$. The supernatant fraction was mixed with a $4 \mathrm{ml}$ volume of Ni-NTA Sepharose resin (Invitrogen, ProBond) equilibrated with a mixture of $50 \mathrm{~m} M$ sodium phosphate, $20 \mathrm{~m} M$ imidazole, $1 \%(w / v)$ Octyl-Glc and $0.3 \mathrm{M} \mathrm{NaCl}$ and gently stirred at $277 \mathrm{~K}$ for $120 \mathrm{~min}$ and packed into two open columns. The columns were washed with $60 \mathrm{ml}$ of buffer containing $50 \mathrm{~m} M$ sodium phosphate, $40 \mathrm{~m} M$ imidazole, $2 \%(w / v)$ Octyl-Glc, $1 M \mathrm{MaCl}$ pH 7.2 and eluted with $14 \mathrm{ml}$ of buffer containing $50 \mathrm{~m} M$ sodium phosphate, $250 \mathrm{~m} M$ imidazole and $2 \%(w / v)$ Octyl-Glc. Fractions containing homogeneous OprM-His $(\mathrm{MW}=51.7 \mathrm{kDa}, 474$ amino acids $)$ were used for crystallization.

\section{Crystallization and crystallographic studies}

Crystal Screen kits (Hampton Research Corp.) were used for initial screening. The setup was kept at $293 \mathrm{~K}$ in an incubator for two to there weeks. The following refinements were subsequently made. The OprM protein was dialyzed against a solution of $50 \mathrm{~m} M$ Tris- $\mathrm{HCl} \mathrm{pH}$ 8.0, $100 \mathrm{~m} M$ imidazole $\mathrm{pH}$ 8.0, 450-600 $\mathrm{m} M$ sodium acetate, $30 \mathrm{mM}$ cyclohexyl-propyl- $\beta$-D-maltoside (CYMAL-3; CMC $=34.5 \mathrm{~m} M$, $\mathrm{MW}=466.5$; Anatrace Inc.), $0.1 \%(v / v) n$-octyl-oligo-oxyethylene (Octyl-POE; $\mathrm{CMC}=6.6 \mathrm{mM}, \mathrm{MW}=400$; Alexis Corp.) and $5 \%(v / v)$ 2-methyl-2,4-pentanediol (MPD; Wako Pure Chemical Industries Ltd) for $6 \mathrm{~h}$ by changing the dialysis buffer every $2 \mathrm{~h}$ and was concentrated to about $12 \mathrm{mg} \mathrm{ml}^{-1}$ protein using a Vivaspin concentrator (VivaScience). The hanging-drop vapour-diffusion technique 


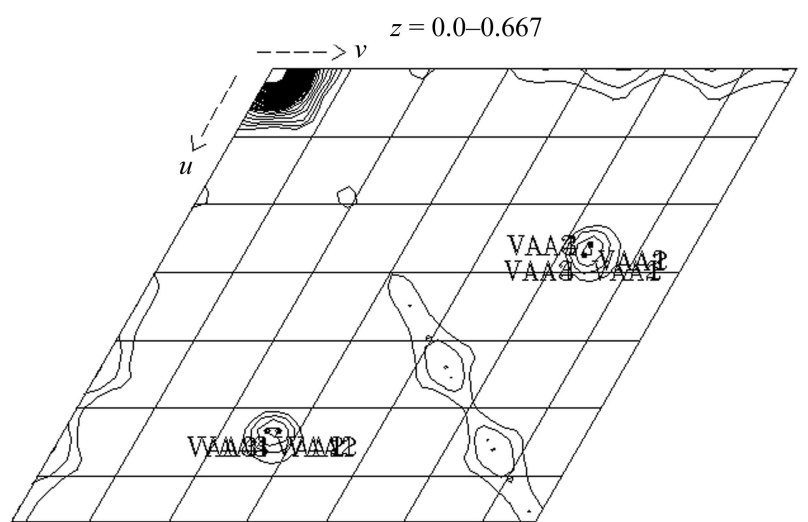

(a)

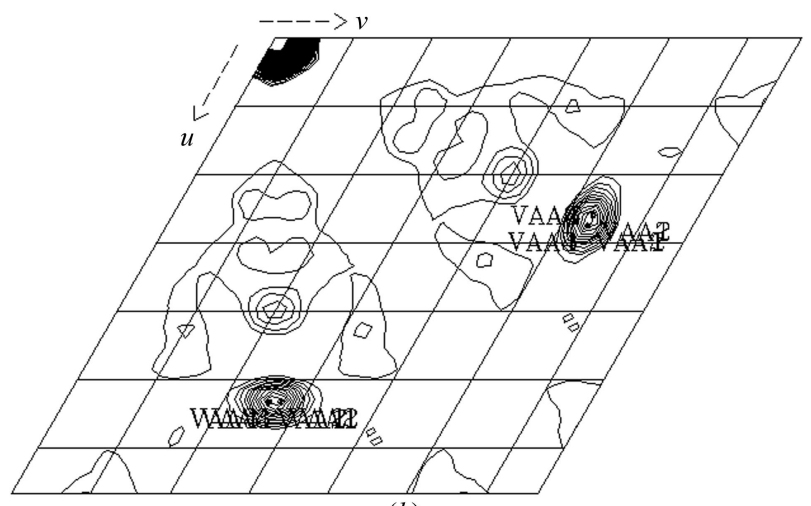

(b)

Figure 2

Difference Patterson map in the Harker plane. (a) shows the isomorphous difference map and $(b)$ the Bijvoet anomalous difference map. Both peaks are from atomic vector site $A(x, y, z)=(0.0645,0.4028,0.3220)$. The maps were contoured at intervals of 1.0 times the r.m.s. density of the map.

was used throughout screening and optimization. The reservoir contained $0.5 \mathrm{ml}$ of the same buffer used for dialysis except that the MPD concentration was varied from 22 to $27 \%(v / v)$. Rhombohedral crystals grew to dimensions of $0.8 \times 0.4 \times 0.2 \mathrm{~mm}$ in a few weeks (Fig. 1). The crystals were flash-cooled in an $\mathrm{N}_{2}$-gas stream at $95 \mathrm{~K}$ and stored in liquid nitrogen for further data collection.

Diffraction data of native OprM and of the iridium derivative were collected at the synchrotron-radiation source at SPring-8 (Hyogo, Japan) using a DIP6040 imaging-plate detector (Bruker AXS/MAC Science) at $95 \mathrm{~K}$. For data collection, the crystal-to-detector distance, oscillation range and exposure time were set to $550 \mathrm{~mm}, 1^{\circ}$ and $30 \mathrm{~s}$ per frame, respectively. The space group of the native crystal was $R 32$, with unit-cell parameters $a=b=85.43, c=1044.3 \AA, \alpha=\beta=90$, $\gamma=120^{\circ}$. The diffraction images were reduced, scaled and merged with the programs DENZO and SCALEPACK (Otwinowski \& Minor, 1997) or MOSFLM (Leslie, 1992) and SCALA (Kabsch, 1988). The intensities were then converted to structure-factor amplitudes with TRUNCATE from CCP4 (Collaborative Computa- tional Project, Number 4, 1994). A set of X-ray diffraction data was collected to $2.56 \AA$ resolution from crystal Native-1. A summary of the crystallographic data is given in Table 1 . The presence of two OprM-His $_{6}$ monomer molecules per asymmetric unit gave a crystal volume per protein weight $\left(V_{\mathrm{M}}\right)$ of $3.5 \AA^{3} \mathrm{Da}^{-1}$ and a solvent content of $65.1 \%$ by volume.

The single isomorphous replacement with anomalous scattering (SIRAS) method using the iridium-derivative crystal was applied to solve the crystallographic phase problem. Native-2 data were used as the native data set for phase determination. The heavy-atom derivative was obtained by soaking the crystal in $2 \mathrm{mM} \mathrm{IrCl}_{3}$ for $16 \mathrm{~h}$. A difference Patterson map of Native- 2 and iridium-derivative data showed clear peaks corresponding to the heavy atom-heavy atom vectors (Fig. 2). The self-rotation function map only shows a clear peak at $(\varphi, \psi, \kappa)=(0.0,0.0,120.0)$, which corresponds to crystallographic threefold symmetry. Since OprM is expected to be a trimeric structure, as in the AcrB and TolC structures, it is assumed that the trimeric structure of OprM is located at the crystallographic threefold axes in the crystal. The unusually long $c$ axis is caused by the complex lattice in the hexagonal setting $R 32$ and the trimeric structure of OprM on the crystallographic threefold axis. Further refinement of the heavy-atom parameters and phasing are in progress.

This work was performed under the Cooperative Research Program of the Institute for Protein Research, Osaka University. The authors are grateful to the beamline staff of SPring8 BL44XU (E. Yamashita and M. Yoshimura). This work was supported in part by grants from the Ministry of Education, Culture, Sport, Science and Technology for the 21st Century Centres of Excellence Research and the Protein 3000 project and from Tokai University (Project Research Grant) and Tokai University School of Medicine (Research Project Grant).

\section{References}

Akama, H., Matsuura, T., Kashiwagi, S., Yoneyama, H., Tsukihara, T., Nakagawa, A., Narita, S. \& Nakae, T. (2004). J. Biol. Chem. 279, 2593925942.

Collaborative Computational Project, Number 4 (1994). Acta Cryst. D50, 760 763.

Higgins, M. K., Bokma, E., Koronakis, E., Hughes, C. \& Koronakis, V. (2004). Proc. Natl Acad. Sci. USA, 101, 9994-9999.

Kabsch, W. (1988). J. Appl. Cryst. 21, 916-924.

Koronakis, V., Sharff, A., Koronakis, E., Luisi, B. \& Hughes, C. (2000). Nature (London), 405, 914-919.

Leslie, A. G. W. (1992). Jnt CCP4/ESF-EAMCB Newsl. Protein Crystallogr. 26.

Murakami, S., Nakashima, R. \& Yamaguchi, A. (2002). Nature (London), 419, 587-593.

Nakajima, A., Sugimoto, Y., Yoneyama, H. \& Nakae, T. (2000). J. Biol. Chem. 275, 30064-30068.

Otwinowski, Z. \& Minor, W. (1997). Methods Enzymol. 276, 307-326.

Pos, K. M., Schiefner, A., Seeger, M. A. \& Diederichs, K. (2004). FEBS Lett. 564, 333-339. 